\title{
Nuevos registros de Puya (Bromeliaceae) en el departamento de Lambayeque, Perú
}

\author{
New records of Puya (Bromeliaceae) in the department of Lambayeque, Peru
}

\author{
José E. Ayasta * \\ https://orcid.org/0000-0003-1832-1315 \\ ayastae@gmail.com \\ Ana M. Juarez \\ https://orcid.org/0000-0002-5179-4942 \\ juarezii@gmail.com \\ Josefa Escurra \\ https://orcid.org/0000-0002-9459-9661 \\ jescurra@unprg.edu.pe

\section{*Corresponding author} \\ Universidad Nacional Pedro Ruiz Gallo, Herbario PRG. \\ Ciudad Universitaria, Juan XXIII 391, Lambayeque, \\ Perú.

\section{Citación} \\ Ayasta JE, Juarez AM, Escurra J. 2021. Nuevos re- \\ gistros de Puya (Bromeliaceae) en el de- \\ partamento de Lambayeque, Perú. Revista \\ peruana de biología 28(2): e18115 (Mayo \\ 2021). doi: http://dx.doi.org/10.15381/rpb \\ v28i2.18115
}

$\begin{array}{ll}\text { Presentado: } & 05 / 07 / 2020 \\ \text { Aceptado: } & 07 / 01 / 2021 \\ \text { Publicado online: } & \text { 25/05/2021 }\end{array}$

Editor:

Leonardo Romero

\section{Resumen}

Este estudio proporciona una lista comentada de las especies de Puya (Bromeliaceae) registradas en el departamento de Lambayeque, Perú. Se reportan un total de cinco especies, de las cuales cuatro se registran por primera vez y una de ellas se confirma como endemismo de los Andes del Departamento. Las ecorregiones donde se encuentran corresponden al Bosque Seco estacional (BSe), la transición Matorral Desértico-Bosque Seco (MD-BS) y Jalca (JA). Se presenta una clave para las especies reportadas y se discuten las razones de los endemismos, amenazas y sus criterios de categorización.

\section{Abstract}

This study provides an annotated checklist of the Puya (Bromeliaceae) species registered from Lambayeque Department; Peru. A total of five species are reported, four are registered for the first time and one of them is confirmed as endemism of Lambayeque Andes. Species were from the seasonal Dry Forest (BSe), Desert Scrub-Dry Forest transition (MD-BS) and Jalca (JA) ecoregions. A key for the Puya reported species is presented and the reasons for endemisms, threats and their categorization criteria are discussed.

Palabras clave:

Puya, Puyopsis, Bromeliaceae, endemismos, flora de Lambayeque.

Keywords:

Puya, Puyopsis, Bromeliaceae, endemism, flora of Lambayeque.

\section{Introducción}

Puya (Bromeliaceae) es un género con más de 200 especies (Gouda \& Butcher 2020), fue descrito por Molina, en base a Puya chilensis (Grant \& Zijlstra 1998), inicialmente incluido por Smith y Downs (1974) en la subfamilia Pitcairnioideae, posteriormente separado de esta subfamilia por su cercanía filogenética con Bromelioideae (Terry et al. 1997) y ubicado por Givnish et al. (2007) en la subfamilia Puyoideae. Se distribuye desde el centro de Chile y Argentina hacia la región andina, teniendo el mayor número de especies en las elevaciones y latitudes medias al sur del Ecuador (Jabaily \& Sytsma 2012, Madriñán 2015) llegando a registrarse algunas especies en Centroamérica (Morales 2003) y ocupando una diversidad de hábitats húmedos y secos con manifestaciones adaptativas muy particulares (Grau et al. 2010, Yepez 2017), como por ejemplo los relacionados con polinizadores específicos (Kessler et al. 2020, Salinas et al. 2007, Velásquez-Noriega et al. 2020, Hornung-Leoni et al. 2013a, Givnish et al. 2014, RestrepoChica \& Bonilla-Gómez 2017, García-Meneses 2012). 
Smith y Downs (1974) subdividieron el género en dos subgéneros de acuerdo con la composición de sus inflorescencias: el subgénero Puya, con las ramas de sus inflorescencias estériles en su parte apical, característica que según Anderson et al. (2005) posiblemente sea una adaptación a la polinización por perchas; y el subgénero Puyopsis, con las ramas fértiles a todo lo largo del eje. Estos criterios de clasificación se han mantenido hasta la actualidad; sin embargo, Jabaily y Sytsma (2010), sostienen que se evidencian pocos estudios sistemáticos a nivel infragenérico dentro de Puya debido a que muchas especies se conocen solo por tipos y por otro lado los caracteres para las comparaciones morfológicas no se conservan en la mayoría de los especímenes de herbario.

En el Perú, Mc Bride (1936) fue el primero en describir 29 especies de Puya; años más tarde se complementan con las descripciones de Smith y Downs (1974) y el registro de 73 especies expuesta por Brako y Zarucchi (1993); asimismo, Hornung-Leoni y Sosa (2006), incluyen, en su estudio alométrico de la variación morfológica de Puya, 18 especies colectadas en diferentes departamentos del Perú y registradas en diferentes herbarios, el cual es complementado por el estudio morfológico y filogenético de Puya subgénero Puya, donde se analizan igualmente especies peruanas (Hornung-Leoni \& Sosa 2008). Posteriormente León et al. (2006) mencionan a más de 60 especies de Puya endémicas del Perú basada en registros de herbario, muchos de los cuales por falta de datos de poblaciones no se les asigna una categoría de amenaza.

Asimismo, son numerosos los trabajos que señalan estudios sobre las poblaciones, usos y estado de conservación de la especie más representativa del género: Puya raimondii (Aquino et al. 2018a, Montesinos 2014, Hornung-Leoni et al. 2013b, Salazar-Castillo \& VillasanteBenavides 2012, Salazar-Castillo, et al. 2010, HornungLeoni \& Sosa 2004) y además existe el registro de otras especies como parte de la flora de diferentes ecosistemas, que van desde las lomas de poca elevación en los departamentos de La Libertad y Lima (Dillon et. al. 2011, Lleellish et al. 2015, Cano et al. 1999), hasta los valles interandinos secos, jalca y puna (Santa Cruz et al. 2019, Montesinos-Tubée et al. 2015, Aquino et al. 2018b). También, en los últimos años se han descrito nuevas especies para el sur del Perú: Puya cahuachensis (Galán de Mera et al. 2016); Puya hoxeyi (Janeba 2017) y Puya colcaensis (Treviño-Zeballos et al. 2019) lo que denota su amplia diversidad, la cual necesita estudiarse con más profundidad.

Antes de este trabajo, en el departamento de Lambayeque, del género Puya sólo era conocida una especie endémica de los pajonales altoandinos de la Provincia de Ferreñafe, basada en una colección única de Ramón Ferreyra (Smith, 1990; Brako \& Zarucchi 1993, León et al. 2006), existiendo un vacío de información sobre la distribución del género en esta zona del Perú, así como su estado de conservación, razones por lo que en este trabajo se amplía la revisión y se proporcionan claves para la identificación de las especies.

\section{Material y métodos}

Área de estudio.- El área de estudio comprende el ámbito del distrito de Incahuasi, (6³'58", 6²3'42"S y 79¹2'68', 79³2'74”'W) en la provincia de Ferreñafe, departamento de Lambayeque, entre los $230 \mathrm{~m}$ y $4060 \mathrm{~m}$, colindante al este con las provincias de Chota y Cutervo, Cajamarca. Geográficamente esta área pertenece a la microcuenca del rio Moyán, desde sus nacientes en la laguna altoandina Tembladera, discurriendo en dirección suroeste hasta su confluencia con el rio Sangana, dando lugar a la formación del rio La Leche como parte de la cuenca del Pacífico (PRONAMACHCS 2000). La zona de estudio se encuentra incluida en la región biogeográfica Amotape-Huancabamba (Weigend 2002), siendo características las formaciones que van desde las escarpadas laderas cubiertas de árboles caducifolios y vegetación xerófita del Bosque Seco Estacional (BSe), la transición Matorral Desértico-Bosque Seco (MD-BS) con vegetación baja adaptada a condiciones de aridez, la vegetación perennifolia de Bosque Pluvial Montano Noroeste (BPMN) y el graminétum andino de la Jalca (JA) alternado con pequeños arbustos (Ayasta \& Juarez 2020).

Metodología.- Se revisó la colección de Puya del departamento de Lambayeque, depositadas en el herbario de la Universidad Nacional Pedro Ruiz Gallo (PRG), así como la comparación con especímenes del Herbario de la Universidad Nacional Mayor de San Marcos (USM) y de catálogos virtuales y bases de datos de Field Museum [https://plantidtools.fieldmuseum.org/es/rrc/searchresults/puya], New York Botanical Garden [http:// sweetgum.nybg.org/science/vh/taxon-checklist], United States National Herbarium [https://collections. nmnh.si.edu/search/botany], Missouri Botanical Garden [http://www. tropicos.org/] y Digital specimen images at the Herbarium Berolinense [http://ww2. bgbm.org/ herbarium/]. Para ubicar los lugares de colecta, se utilizó la clasificación de ecorregiones de Brito (2017), los puntos de colecta fueron debidamente georeferenciados y corresponden a ecosistemas de bosque seco y jalca; estos se presentan en la Figura 1.

La identificación de las especies se realizó inicialmente siguiendo las claves de Smith y Downs (1974) y la revisión final de acuerdo con Gouda, Butcher y Gouda (2020); los nombres considerados para las especies están de acuerdo a Gouda y Butcher (2020) y The Plant List (2013) y los acrónimos de herbario según el Index Herbariorum (NYBG 2020, en línea). Asimismo, el procesamiento, dibujos de estructuras y determinación de las especies se realizó en el Herbario PRG, donde se encuentra toda la colección de los autores.

\section{Resultados y discusión}

Se registraron cinco especies de Puya Molina, todas ellas pertenecientes al subgénero Puyopsis (Gouda, Butcher \& Gouda 2020); de estas, cuatro se registran por primera vez para el departamento de Lambayeque (Tabla 1), mientras que, hasta el momento, tres especies son endemismos del Perú y una endémica de los Andes del departamento de Lambayeque (Smith, 1990, León et al. 
2006). De acuerdo con la distribución altitudinal y el ecosistema donde se encuentran, cada una de las especies se ubica en una sola ecorregión es decir su distribución se restringe a una pequeña área, con poblaciones de pocos individuos en un solo ecosistema. Puya ferruginea es la especie que se distribuye en el rango altitudinal más bajo en el BSe (900-1000 m), en zonas rocosas, escarpadas y de difícil acceso; en el rango altitudinal medio se encuentra Puya glaucovirens, en laderas secas e intervenidas sobre los $2000 \mathrm{~m}$ del MD-BS (Fig. 2) y las otras tres especies ocupan una sola área de Jalca (JA), sobre los 4000 m, intercalándose con la vegetación herbácea propia de esta ecorregión.

Estas tres ecorregiones forman parte de la región biogeográfica Amotape-Huancabamba (Weigend 2002), por lo que se comparten con los departamentos adyacentes de La Libertad y Cajamarca, donde se han registrado también las especies Puya ferruginea (Dillon et. al. 2011, Beltrán et al. 2017, Hornung-Leoni \& Sosa 2008), Puya glaucovirens (Santa Cruz et al. 2019), Puya isabellina (León et al. 2006) y Puya hamata (Hornung-Leoni \& Sosa 2006) ésta última presente también en las Jalcas y Páramos de Ecuador y Colombia (García-Meneses \& Ramsay 2014, Mandriñán 2015, Jabaily \& Sytsma 2010). Se confirma en este estudio a Puya ramonii como endemismo del departamento de Lambayeque.

\section{Taxonomía}

\section{Puya Molina}

Saggio Chili 160, 351. 1792. Tipo: Puya chilensis.

Descripción: Hierbas perennes, generalmente largamente-caulescentes; caule robusto formado por la vaina de las hojas lignificadas; simples o ramificadas, robustas, frecuentemente con inflorescencias de varios metros de alto o cortamente cilíndricas. Hojas densamente rosuladas, coriáceas; vainas distinguibles, generalmente amplias; láminas triangulares casi siempre armadas de aguijones ganchosos. Inflorescencia simple paniculada; flores vistosas. Sépalos convolutos, libres, mucho más cortos que los pétalos, glabros, tomentosos o lanados; pétalos libres, generalmente con láminas amplias, extendidas en la antesis y después dispuestas en espiral; estambres generalmente más cortos que los pétalos; filamentos libres. Ovario súpero o semiínfero, glabro; estilo largo y delgado, cápsula loculicida y en general tardíamente septicida. Semillas largas con un ala dorsal y apical.

Es un género distribuido principalmente en los páramos y regiones xerofíticas altas, desde Costa Rica a Chile. Etimológicamente debe su nombre al vocablo araucano "puya", que significa punzante, haciendo referencia a las puntas y bordes de las hojas.
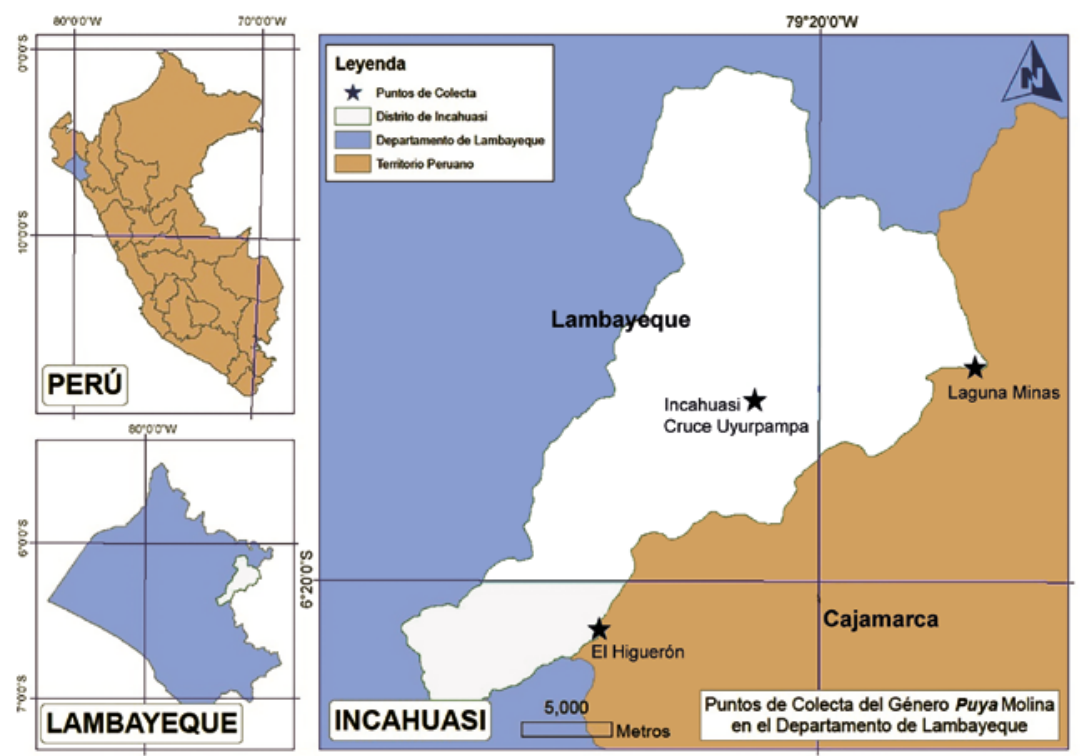

Figura 1. Zonas de colecta de Puya en el área de estudio (Distrito de Incahuasi, Provincia de Ferreñafe, Lambayeque).

Tabla 1. Registro de Puya en el departamento de Lambayeque, Perú. Los Endemismos para el Perú, están de acuerdo a León et al. (2006) y las Ecorregiones están basadas en Britto (2017): BSe: Bosque Seco estacional; MDE-BS: Matorral desértico-Bosque Seco; JA: Jalca. NE: no endémico; ENP: Endémico del norte de Perú; ELa: Endémico de Lambayeque.

\begin{tabular}{|c|c|c|c|c|}
\hline Especie & Endemismo & Referencias y Reportes (Lambayeque-Perú) & Altitud (m) & Ecorregión \\
\hline Puya ferruginea (Ruiz y Pav.) L.B. Sm. & NE & Nuevo Reporte & $900-1000$ & BSe \\
\hline Puya glaucovirens Mez. & ENP & Nuevo Reporte & $2000-2500$ & MDE-BS \\
\hline Puya hamata L.B.Smith & NE & Nuevo Reporte & 4000 & $\mathrm{JA}$ \\
\hline Puya isabellina Mez & ENP & Nuevo Reporte & 4000 & $J A$ \\
\hline Puya ramonii L.B.Smith & ELa & $\begin{array}{l}\text { Colectada por Ferreyra, } 1987 \text { (Tipo } 20910 \text { USM). } \\
\text { Reportada como especie nueva por Smith (1990) }\end{array}$ & 4000 & $J A$ \\
\hline
\end{tabular}


Clave para las especies de Puya registradas en Lambayeque:

1. Últimas ramas de la inflorescencia visibles en la antesis. Inflorescencias delgadas, no estrobiliformes. Brácteas florales foliáceas $\mathbf{2}$

$1^{\prime}$. Últimos ejes de la inflorescencia completamente cubiertos. Inflorescencia estrobiliforme. Brácteas florales coriáceas.

2. Inflorescencias cubiertas por una indumentaria ferrugínea. Bráctea floral ovada-triangular con el ápice redondeado. Puya ferruginea

2'. Inflorescencias cubiertas con escamas escábridas, verdes. Bráctea floral anchamente ovada, con el ápice agudo. Puya glaucovirens

3. Brácteas exteriores con sus contornos cubiertos por el indumento lanoso denso. Brácteas florales agudas o acuminadas. Vainas de las hojas amplias, semicirculares, fuertemente cóncavas. Puya hamata

3'Brácteas exteriores con sus contornos claramente definidos; contorno no cubierto por el indumento lanoso.

4

4. Inflorescencia ramificada, con sus ramas algo curvadas, densas. Puya ramonii

4’. Inflorescencia simple, cilíndrica, casi cónica. Puya isabellina

\section{Puya ferruginea (Ruiz \& Pav.) L.B. Smith}

Phytologia 16(6): 461 (1968).

Tipo: Ruiz \& Pavón. s n (Holotipo BM), Amancaes, Lima, Perú.

Distribución: Especie reportada desde el sur de Ecuador, Perú y Bolivia, entre los 1800 y 3800 m. En el Perú se ha reportado en varios departamentos (Brako \& Zarucchi, 1993, Hornung-Leoni \& Sosa 2008) en zonas áridas y pendientes de difícil acceso, así como también asociada a vegetación de lomas (Dillon et al. 2011, Lleellish 2015, Cano et al. 1999). En la zona de estudio se le encuentra en rocas desnudas fuertemente escarpadas, en asociación con Tillandsia rauhii y vegetación herbácea xerófita, entre los $900 \mathrm{~m}$ y $1000 \mathrm{~m}$ en el bosque seco estacional (BSe).

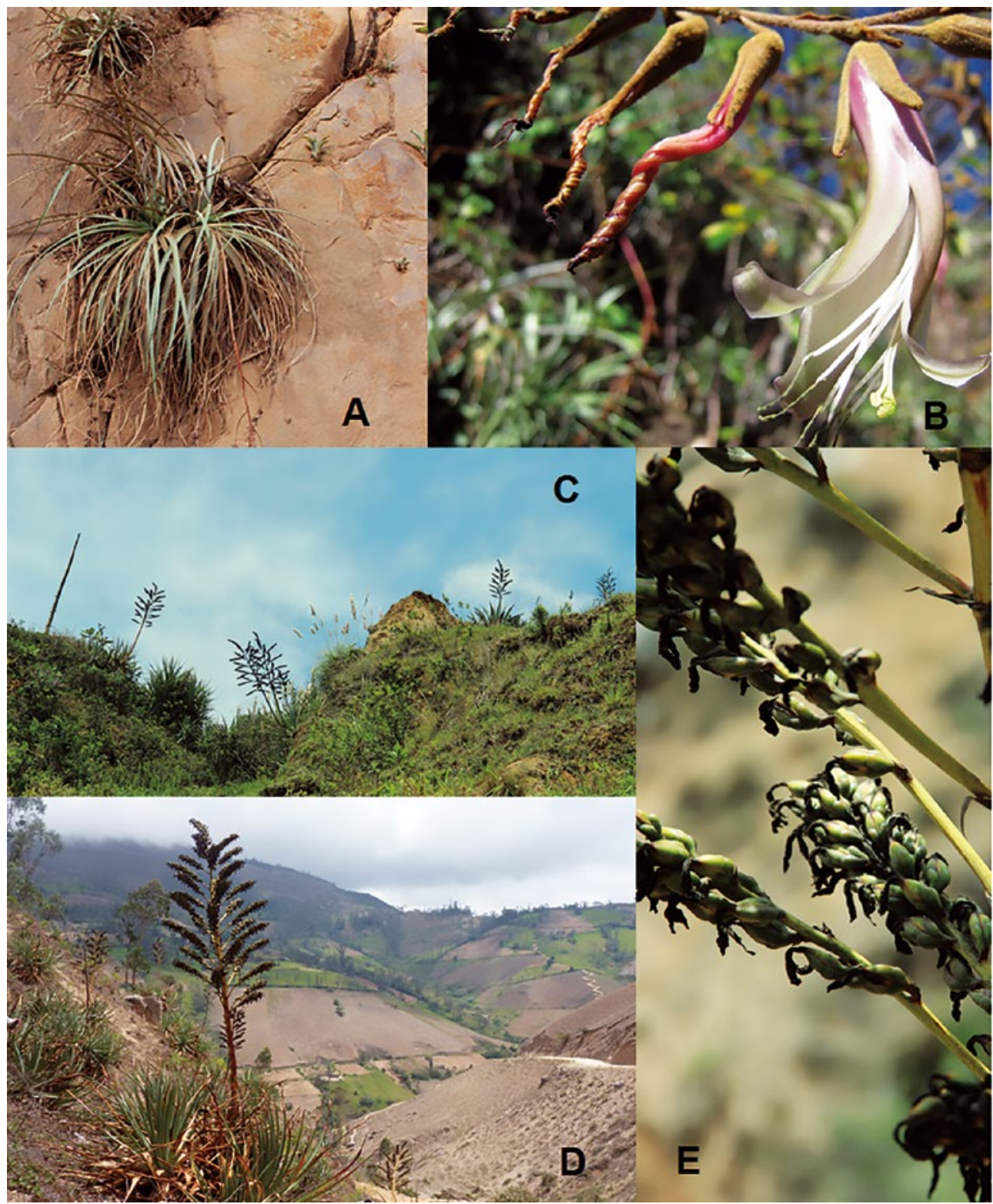

Figura 2. A-B. Puya ferruginea. A. Hábito. B. Inflorescencia y flor abierta. C-E. Puya glaucovirens. C. Comunidad en época húmeda. D. Individuo en ladera intervenida. E. Detalle de las ramas de la Inflorescencia. 
Conservación: Esta especie ha sido colectada en la zona de amortiguamiento del Área Natural Protegida Refugio de Vida Silvestre Laquipampa; por las características de hábitat casi inaccesible podríamos decir que no se encuentra amenazada, lo que de alguna manera asegura su conservación; sin embargo, su población no es tan numerosa.

Material de referencia: PERU: Departamento de Lambayeque, Provincia de Ferreñafe. Distrito de Inkawasi, Higuerón-Laquipampa, 6²1'29.44"S, 79²6'34.86”W, 900 m, 26 Febrero 2009, J. Ayasta y A. Juarez. 15083 PRG (Fig. 3).

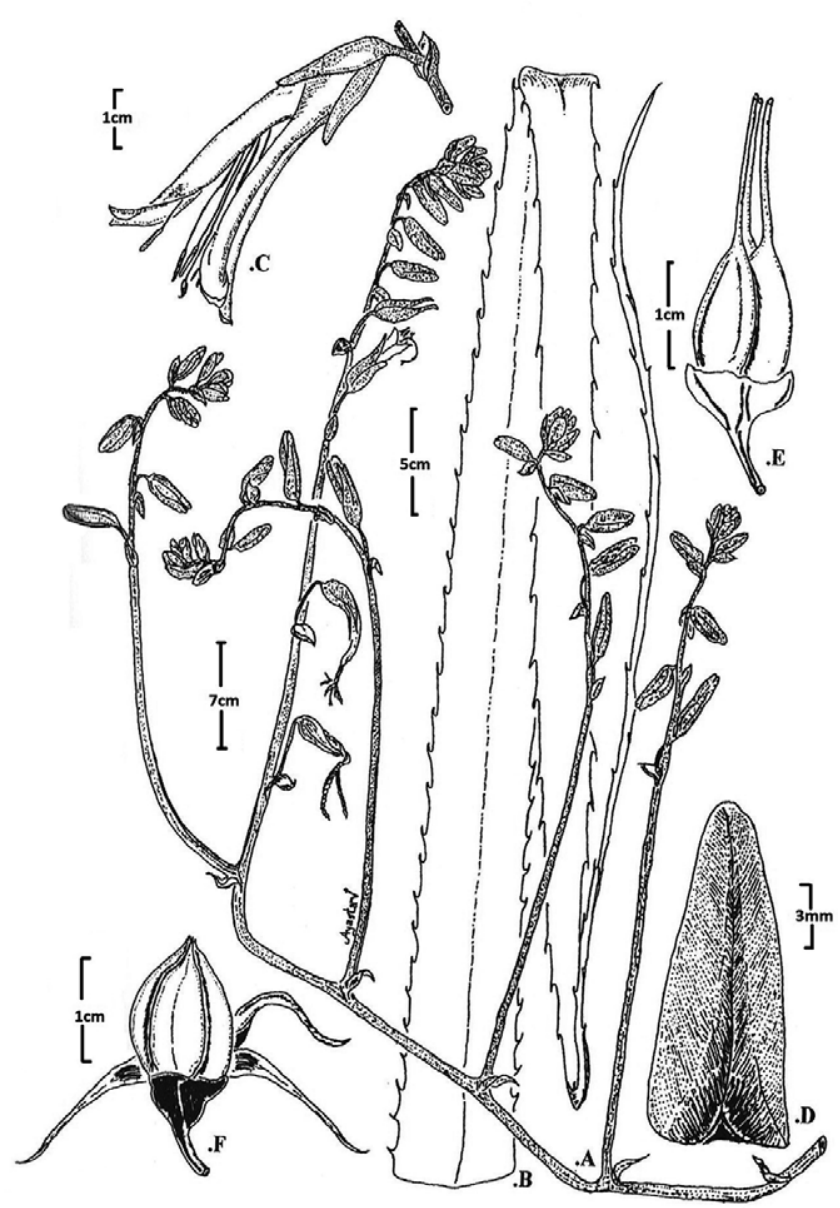

Figura 3. Puya ferruginea (Ruiz \& Pav.) L.B. Smith. A. Inflorescencia. B. Hoja. C. Flor. D. Bráctea Floral. E. Ovario. F. Fruto y cáliz (dibujado de J. Ayasta y A. Juarez. 15083 PRG).

\section{Puya glaucovirens $\mathrm{Mez}$}

Repertorium Specierum Novarum Regni Vegetabilis 3: 9.1906.

Tipo: Weberbauer 4208 (Holotipo B. Fototipo F). Huambos, Chota, Cajamarca, Perú, 31 May 1904.

Distribución: Endémica de los andes norperuanos; hasta hoy se reporta solamente en los departamentos de Cajamarca y Amazonas (Gouda, Butcher \& Gouda 2020). En la zona de estudio se le encuentra en áreas secas y templadas, entre los $2000 \mathrm{~m}$ y $2500 \mathrm{~m}$ en la transición Matorral Desértico-Bosque Seco (MD-BS).
Conservación: En el área de estudio, esta especie no se encuentra en ninguna Área Natural Protegida. Se han observado pocos individuos a lo largo de las laderas secas en el margen del Rio Moyán-Incahuasi y está amenazada principalmente por la quema de sus individuos para generar áreas por el avance de la agricultura y la introducción de especies exóticas (áreas deforestadas y quemadas para la siembra de Eucalyptus). Según los criterios de categorización de especies amenazadas UICN 2017, podría considerarse esta especie para el Perú, como una especie vulnerable: VU B2 b(iii); si bien es cierto se encuentra en Amazonas, Cajamarca y Lambayeque, el área de ocupación de las poblaciones no es muy extensa y está sometida a la disminución contínua de la calidad del hábitat.

Material de referencia: PERÚ: Departamento de Lambayeque, Provincia de Ferreñafe. Distrito de Inkawasi. Cruce Uyurpampa. 6¹4'31.38"S 79²1'56.80"0. 2200 m. 26 Agosto 2004. J. Ayasta \& A. Juarez 15061 PRG. Distrito de Inkawasi. Riopampa 6² 14 '23.02"S 79²1'23.07"0 2283 m. 19 Enero 2015. J. Ayasta \& A. Juarez 15315 PRG (Fig. 4).

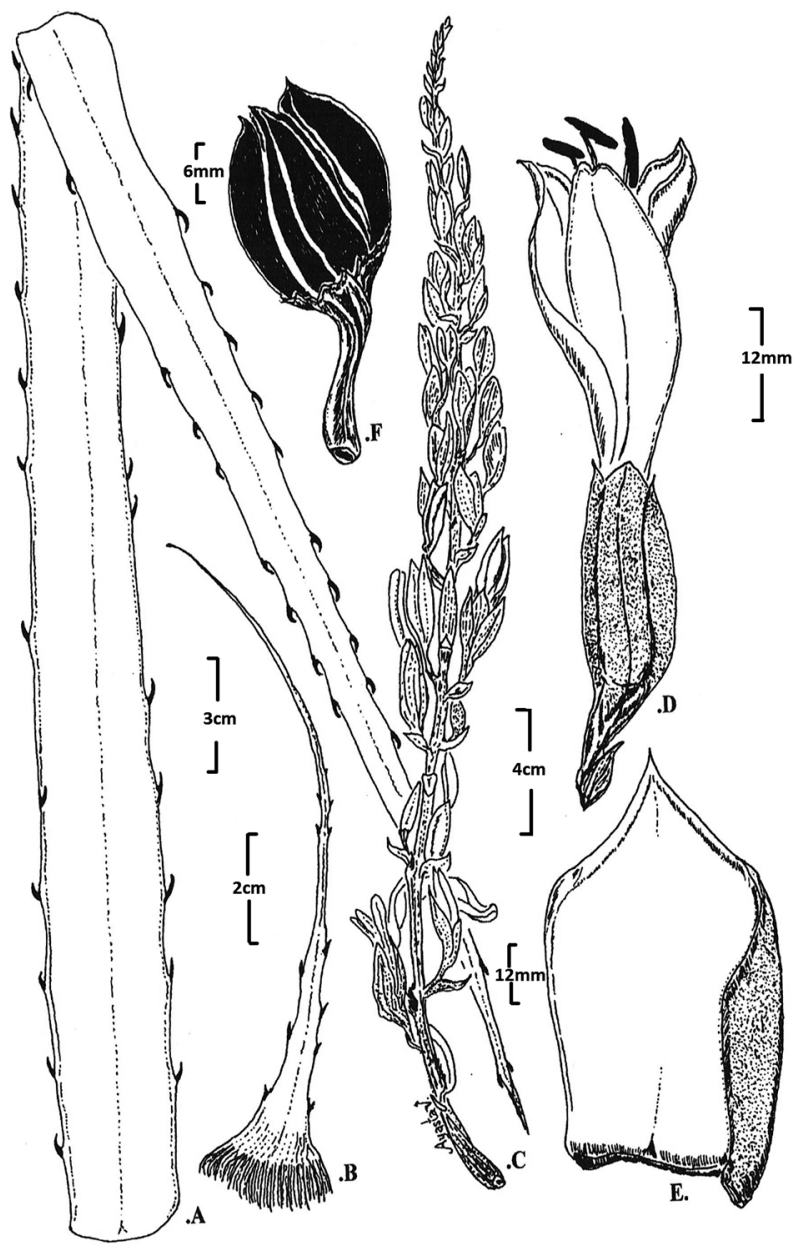

Figura 4. Puya glaucovirens Mez. A. Hoja. B. Bráctea del escapo. C. Rama de la Inflorescencia (inmadura) D. Flor. E. Bráctea Floral. F. Fruto. (dibujado de J. Ayasta y A. Juarez. 15061 PRG). 
Puya hamata L.B.Sm.emended L.B.Sm. \& R.W.Read.

Contrib. U.S. Herb. 29(11): 315 (1949).

Phytologia. 52(1): 49 (1982).

Tipo: Hitchcock 20915 (Holotipo US), 12 millas al oeste de Tulcán, Carchi, Ecuador, 10 Aug 1923.

Distribución: Propia de los páramos del suroeste de Colombia, este de Ecuador (García-Meneses \& Ramsay 2014) y la jalca noroeste del Perú; hasta el momento reportada en el Perú solamente para el departamento de Cajamarca (Smith \& Downs, 1974; Brako \& Zarucchi, 1993, Hornung-Leoni \& Sosa 2006). En la zona de estudio se le encuentra en la Jalca, sobre los $4000 \mathrm{~m}$.

Usos: Las inflorescencias inmaduras de esta especie (junto con las de otras especies recolectadas en Cajamarca), son comercializadas eventualmente en los mercados de Chiclayo con el nombre de "carnero", "lana de carnero" o "hierba de carnero" como medicinal (tranquilizante), lo que aumenta su amenaza de vulnerabilidad por la extracción sin ningún control.

Conservación: Esta especie no se encuentra en ninguna Área Natural Protegida en el área de estudio, encontrándose restringida a una sola población de pocos individuos en zonas rocosas alrededor de la Laguna Minas-Incahuasi.

Material de referencia: PERÚ: Departamento de Lambayeque, Provincia de Ferreñafe. Distrito de Inkawasi. Cerca de la Laguna Minas. 6 14'35.02"'S 79¹3'47.7"O. 4060 m. 25 Agosto 2001. J. Ayasta; A. Juarez; R. Aguirre \& C. Sánchez 15055 PRG (Fig.5).

\section{Puya isabellina Mez}

Repertorium Specierum Novarum Regni Vegetabilis 16: 66. 1919.

Tipo: Weberbauer 4144 sin fecha. Perú: Cajamarca: entre Santa Cruz y Hualgayoc (B; Fototipo F).

Distribución: Se reporta como un endemismo peruano (León et al. 2006); hasta hoy solamente colectado en los departamentos de Amazonas y Cajamarca. En la zona de estudio habita sitios con vegetación propia de jalca, sobre los $4000 \mathrm{~m}$.

Usos: Algunas veces se le encuentra semicultivada como cerco vivo para evitar el paso del ganado ovino y vacuno.

Conservación: La población del departamento de Lambayeque está restringida a pocos individuos que rodean al sitio de colecta y no se registra en ninguna ANP ni está debidamente protegida. Siguiendo los criterios de categorización de especies amenazadas UICN 2017, esta especie podría considerarse para el Perú como una especie En Peligro (EN B2a), ya que si bien es cierto se le registra en Amazonas, Cajamarca y Lambayeque, el área de ocupación es menor a $500 \mathrm{~km}^{2}$, sus poblaciones son pequeñas y su hábitat se encuentra severamente afectado.
Material de referencia: PERÚ: Departamento de Lambayeque, Provincia de Ferreñafe. Distrito de Inkawasi. Cerca de la Laguna Minas. 6 14'31.21's 79¹3'42.3”O. 4060 msnm. 25 Agosto 2001 J. Ayasta; A. Juarez; R. Aguirre \& C. Sánchez 15054 PRG. (Fig. 6).

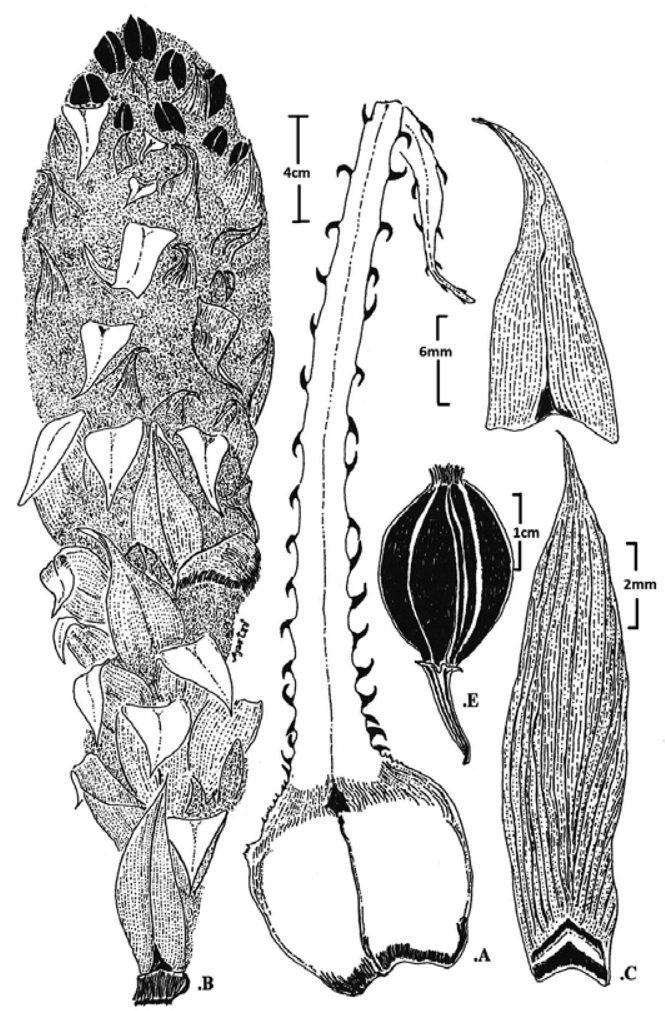

Figura 5. Puya hamata L.B. Smith. A. Hoja. B. Inflorescencia. C. Bráctea Floral. D. Sépalo. F. Fruto. (dibujado de J. Ayasta; A. Juarez; R. Aguirre \& C. Sánchez 15055 PRG).

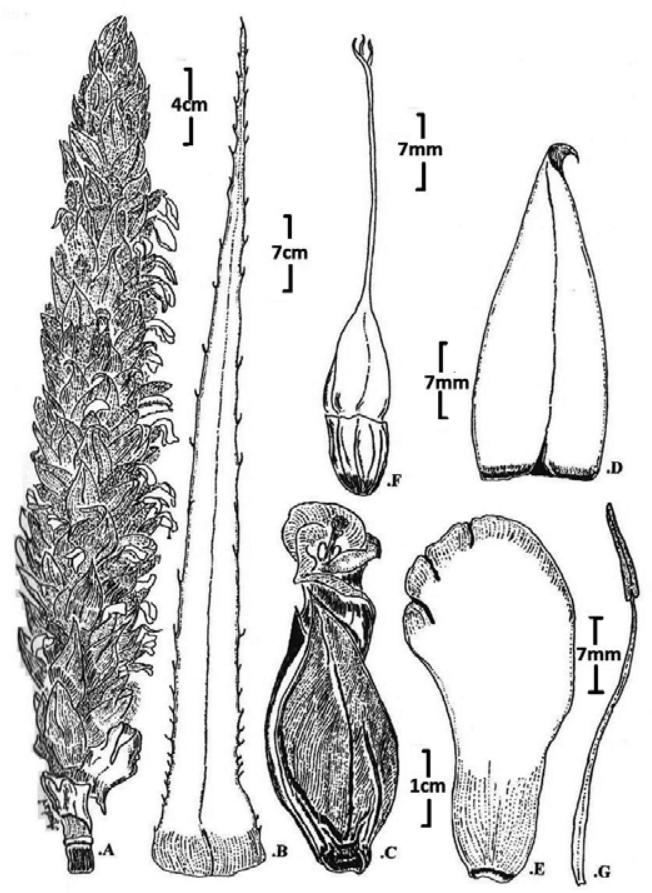

Figura 6. Puya isabellina Mez A. Inflorescencia. B. Hoja. C. Flor. D. Bráctea Floral. E. Pétalo. F. Gineceo. G. Estambre (dibujado de J. Ayasta; A. Juarez; R. Aguirre \& C. Sánchez 15054 PRG). 


\section{Puya ramonii L.B. Smith}

Journal of the Bromeliad Society 40(2): 57. 1990.

Tipo: Perú. Lambayeque: Prov. Ferreñafe; jalca, pajonal. 3700-3800 m. 8/VII/1987. R. Ferreyra 20910 US, (Isotipo USM, PRG).

Distribución: Especie endémica de los andes lambayecanos, colectada por primera vez por Ramón Ferreyra cerca de Incahuasi-Ferreñafe, en 1987. Se confirma en este trabajo su población restringida solamente al departamento de Lambayeque.

Conservación: Debido a la falta de datos para la especie, León et al. 2006, no le asignó una categoría de amenaza; sin embargo, según las observaciones en campo y siguiendo las Directrices de uso de las Categorías y Criterios de la Lista Roja de UICN 2017, la especie tendría la categoría de En Peligro Crítico: CR B1a D, ya que el área de ocupación corresponde a un área menor a 10 $\mathrm{km}^{2}$, con una sola población cuyo número de individuos maduros es menor a 50 .

Material de referencia: PERU: Departamento de Lambayeque, Provincia de Ferreñafe. Distrito de Inkawasi. 3500 msnm. 08 Julio 1987. R. Ferreyra H. 3082471 Holotipo [https://collections.nmnh.si.edu/search/ botany/?qt=Puya+ramonii] 20910 USM Isotipo; 8370 PRG Isotipo. Cerca de la Laguna Minas. 6 $14^{\prime} 31.12^{\prime \prime} \mathrm{S}$ 79¹3'12.3”O 4000 m. 25 Agosto 2001. J. Ayasta; A. Juarez; R. Aguirre \& C. Sánchez 15056 PRG (Fig. 7).

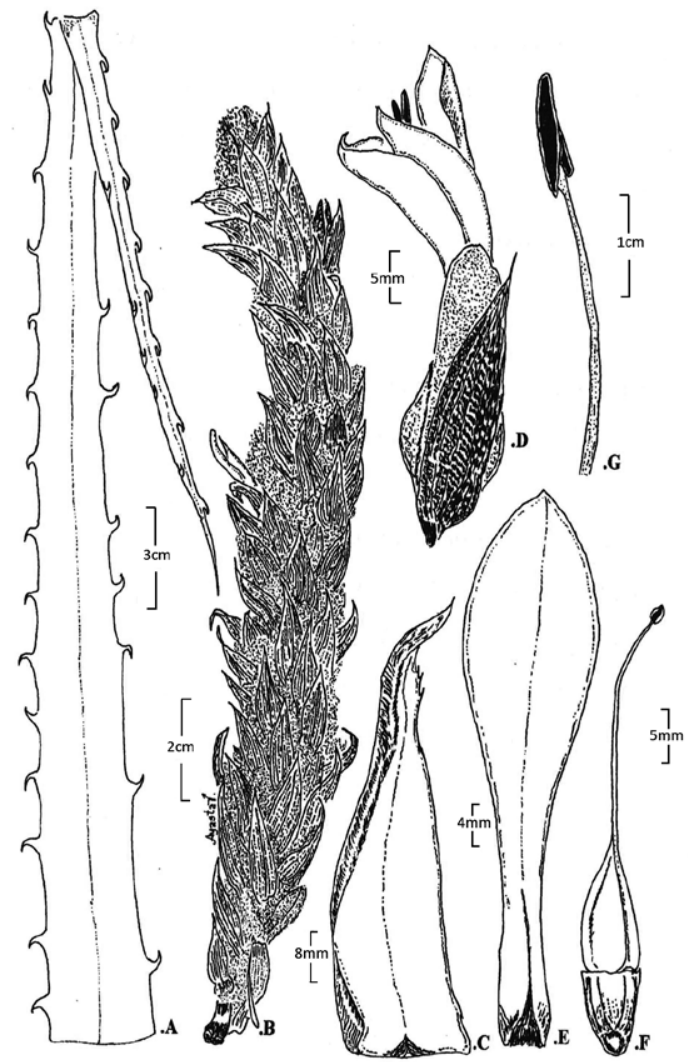

Figura 7. Puya ramonii L. B. Smith. A. Hoja. B. Espiga. C. Bráctea Floral. D. Flor. E. Pétalo. F. Gineceo. G. Estambre (dibujado de . J. Ayasta; A. Juarez; R. Aguirre \& C. Sánchez 15056 PRG).

\section{Literatura citada}

Anderson B, Cole W, Barrett S. 2005. Specialized bird perch aids cross-pollination. Nature 435: 41-42. http://dx.doi. org/10.1038/435041a

Aquino W, Condo F, Romero J, Yllaconza R. 2018a. Distribución geográfica y poblacional de Puya raimondii Harms en el distrito de Huarochirí, provincia de Huarochirí, Lima, Perú. The Biologist 16(1): 25-33.

Aquino W, Condo F, Romero J, Yllaconza, R, La Torre MI. 2018b. Flora y vegetación asociada a los rodales de Puya raimondii de Huarochirí, Lima, Perú. Revista peruana de biología 26(1): 009-020. http://dx.doi.org/10.15381/ rpb.v26i1.14551

Ayasta J, Juarez A. 2020. El género Tillandsia L. (Bromeliaceae) en el departamento de Lambayeque, Perú. Revista Peruana de Biología 27(2): 189-204. http://dx.doi. org/10.15381/rpb.v27i2.15718

Beltrán H. Vadillo-Gálvez G, y Palomino-Zeña F. 2017. Flora y vegetación de la Reserva Nacional de Calipuy, La Libertad. Arnaldoa, 24(1):267-288.

Brako, L. y Zarucchi, J. 1993. Bromeliaceae in: Catalogue of the flowering plants Gymnosperms and Angyosperms of Perú. Monog. Syst. Bot. Miss. Bot. Garden. 45. USA.

Brito B. 2017. Actualización de las Ecorregiones Terrestres de Perú propuestas en el Libro Rojo de Plantas Endémicas del Perú. Gayana Botánica 74(1):15-29. http:// dx.doi.org/10.4067/S0717-66432017005000318

Cano A, Roque J, Arakaki M, Arana C, La Torre M, Llerena N, Refulio N. 1999. Diversidad Florística de las Lomas de Lachay (Lima) durante el Evento "El Niño 1997-98" En El Niño 1997-98 y su Impacto sobre los Ecosistemas Marino y Terrestre. Revista Peruana de Biología Vol. Extraordinario 125-132. https://doi.org/10.15381/ rpb.v6i3.8438

Curators Herbarium B. 2020. Digital specimen images at the Herbarium Berolinense. [Dataset]. Data Publisher: Botanic Garden and Botanical Museum Berlin. http:// ww2.bgbm.org/herbarium/.

Dillon MO, Leiva S, Zapata M, Lezama P. y Quipuscoa V. 2011. Floristic Checklist of the Peruvian Lomas Formations - Catálogo Florístico de las Lomas Peruanas. Arnaldoa 18(1):7-32

Galán de Mera, A., Montoya, J., Vicente Orellana, J. \& Linares Perea, E. 2016. Puya cahuachensis (Bromeliaceae, $\mathrm{Pu}-$ yopsis), a new species from Southern Peru. Phytotaxa 283:286-290. https://doi.org/10.11646/phytotaха.283.3.4

García-Meneses P. 2012. Landscape-scale population dynamics: field observations and modelling of Puya hamata, a flagship plant from the Andes. PhD Thesis, University of Plymouth.

García-Meneses P, Ramsay P. 2014. Puya hamata demography as an indicator of recent fire history in the Paramo of El Ángel and Volcán Chiles, Ecuador-Colombia. Caldasia 36 (1):53-69. https://doi.org/10.15446/caldasia. v36n1.43891

Givnish T, Barfuss M, Van Ee B, Riina R, Schultee K, Horres R, Gonsiskaa P, Jabaily R, Crayn D, Smithi A, Winter K, Brown G, Evans T, Holstm B, Luthern H, Till W, Zizka G, Berry P y Sytsmaa K. 2014. Adaptive radiation, correlated and contingent evolution, and net species diversification in Bromeliaceae. Molecular Phylogenetics and Evolution 71:55-78. https://doi.org/10.1016/j. ympev.2013.10.010 
Gouda E, Butcher D. 2020. A List of Accepted Bromeliaceae Names [http://bromeliad.nl/bromNames/]. University Botanic Gardens, Utrecht (accessed: 28-06-2020).

Gouda E, Butcher D, Gouda C. 2020. Encyclopaedia of Bromeliads, Version 4. http://bromeliad.nl/encyclopedia/ University Botanic Gardens, Utrecht (accessed: 28-062020)

Grant JR, Zijlstra G. (1998). An annotated catalogue of the generic names of the Bromeliaceae. Selbyana, 19(1):91121.

Grau E, Gómez-Romero E, Aráoz E. 2010. Puyas andinas. Ciencia Hoy 20:118.

Hornung-Leoni C, Sosa V. 2004. Uses of the giant bromeliad, Puya raimondii. Journal Bromeliad Society 54: 3-8.

Hornung-Leoni C, Sosa V. 2005. Morphological variation in Puya (Bromeliaceae): an allometric study. Plant Systematics and Evolution 256(1):35-53. https://doi. org/10.1007/s00606-005-0302-z

Hornung-Leoni C, Sosa V. 2008. Morphological phylogenetics of Puya subgenus Puya (Bromeliaceae). Botanical Journal of the Linnean Society 156, 93-110. https://doi. org/10.1111/j.1095-8339.2007.00740.x

Hornung-Leoni C, González-Gómez P, Troncoso A. 2013a. Morphology, nectar characteristics and avian pollinators in five Andean Puya species (Bromeliaceae). Acta Oecologica 51: 54-61 https://doi.org/10.1016/j.actao.2013.05.010

Hornung-Leoni C, Sosa V, Simpson, J y Gil, K. 2013b. Genetic variation in the emblematic Puya raimondii (Bromeliaceae) from Huascarán National Park, Peru. Crop Breeding and Applied Biotechnology, 13(1), 67-74. https:// doi.org/10.1590/S1984-70332013000100008

Index herbariorum. 2020. New York Botanical Garden. http:// sweetgum.nybg.org/science/ih/

Jabaily RS, Sytsma KJ. 2010. Phylogenetics of Puya (Bromeliaceae): Placement, major lineages, and evolution of Chilean species. American Journal of Botany 97(2): 337-356. https://doi.org/10.3732/ajb.0900107

Jabaily RS, Sytsma KJ. 2012. Historical biogeography and lifehistory evolution of Andean Puya (Bromeliaceae). Botanical Journal of the Linnean Society 171:201-224. https://doi.org/10.1111/j.1095-8339.2012.01307

Kessler M, Abrahamczyk S, Kromer T. 2020. The role of hummingbirds in the evolution and diversification of Bromeliaceae: unsupported claims and untested hypotheses. Botanical Journal of the Linnean Society 192:592-608. https://doi.org/10.1093/botlinnean/ boz100

León, B; Sagástegui, A.; Sánchez, I. y Zapata, M. (2006) Bromeliaceae endémicas del Perú. En: El libro rojo de las plantas endémicas del Perú. Ed.: Blanca León et al. Revista Peruana de Biología Número especial 13(2): 708-737. https://doi.org/10.15381/rpb.v13i2.1941

Lleellish M, Odar J, Trinidad H. 2015. Guía de Flora de las Lomas de Lima. Servicio Nacional Forestal y de Fauna Silvestre. Lima, Perú. 162 pag. http://repositorio.serfor.gob. pe/handle/SERFOR/484

Madriñán S. 2015. Una nueva especie de Puya (Bromeliaceae) de los páramos cercanos a Bogotá, Colombia. Revista de la Academia Colombiana de Ciencias Exactas, Físicas y Naturales 39(152):389-398. https://doi. org/10.18257/raccefyn.223

Mc Bride F. 1936. Bromeliaceae in Flora of Peru. Part. I. N 3. Vol. XIII. Field Museum of Natural History. Chicago.
Montesinos D. 2014. Inventario y estado de conservación de Puya raimondii (Bromeliaceae) en el departamento de Moquegua, Perú. Chloris Chilensis (17)1: 1-9. https:// edepot.wur.nl/330210

Montesinos-Tubée D, Cleef A, Sykora K. 2015. The Puna vegetation of Moquegua, South Peru: Chasmophytes, grasslands and Puya raimondii stands. Phytocoenologia 45(4):365-397. https://doi.org/10.1127/ phyto/2015/0006

Morales JF. 2003. Bromeliaceae. In, Hammel BE, Grayum M, Herrera C, Zamora N. (Eds.) Manual of Plants of Costa Rica. vol. II. Monographs in Systematic Botany from the Missouri Botanical Garden. St. Louis 92: 297-375.

PRONAMACHCS (Proyecto Nacional de Manejo de Cuencas Hidrográficas y Conservación de Suielos). 2000. Microcuenca Moyan Inkawasi. Tomo I. Diagnóstico. Ministerio de Agricultura-Perú.

Restrepo-Chica M, Bonilla-Gómez MA. 2017. Dinámica de la fenología y visitantes florales de dos bromelias terrestres de un páramo de Colombia. Revista Mexicana de Biodiversidad 88: 636-645. https://doi. org/10.1016/j.rmb.2017.07.008

Salazar-Castillo J, Cáceres F, Poma I, Raimondo F. 2010. Diagnóstico del estado actual de conservación de Puya raimondii en Arequipa-Perú. Quaderni di Botanica ambientale e applicata 21: 83-91.

Salazar-Castillo J, Villasante-Benavides F. 2012. Distribución geográfica y situación actual de Puya raimondii Harms en la Región de Arequipa - Perú. Octubre 2009 - Marzo 2011. Quaderni di Botanica ambientale e applicata 23: 31-39.

Salinas L, Arana C, Suni M. 2007. El néctar de especies de Puya como recurso para picaflores Altoandinos de Ancash, Perú. Revista Peruana de Biología 14(1): 129-134. https://doi.org/10.15381/rpb.v14i1.2166

Santa Cruz L, Cano A, La Torre M, Rodríguez E, Campos J. 2019. Inventario de la flora de angiospermas del distrito de Pulán, provincia de Santa Cruz, Cajamarca-Perú. Arnaldoa 26 (1): 139-212.

Smith L, Downs R. 1974. Pitcairnoideae. (Bromeliaceae). Flora Neotropica, Monograph14 (1):1-658. The New York Botanical Garden.

Smith L. 1990. A new species for an old friend: Puya ramonii Journal of the Bromeliad Society 40 (2):57-58

Terry R, Brown G, Olmstead G. 1997. Examination of subfamilial phylogeny in Bromeliaceae using comparative sequencing of the plastid locus ndhF. Amer. J. Bot. 84: 664-670. https://doi.org/10.2307/2445903

The Plant List (2013). A working list of all species. http://www. theplantlist.org

Treviño-Zevallos I, Quipuscoa-Silvestre V y Gouda E. 2019. Puya colcaensis (Bromeliaceae) a new species from southern Peru. Phytotaxa 406 (4):237-242. https:// doi.org/10.11646/phytotaxa.406.4.2

UICN 2017. Directrices de Uso de las Categorías y Criterios de la Lista Roja de la UICN. Versión 13. Preparado por el Comité de Estándares y Peticiones UICN. Disponible en http://www.iucnredlist.org/documents/RedListGuidelines.pdf

Velásquez-Noriega P, Mayta C, Cuba E, García E, Montaño-Centellas F, Thorsten K. (2020). Floral ecology and floral visitors of Puya atra (Bromeliaceae), a Bolivian endemic plant. Ecología en Bolivia 55(1):36-45. 
Weigend M. 2002. Observations on the Biogeography of the Amotape-Huancabamba Zone in Northern Peru. The Botanical Review 68:38-54. https://doi. org/10.1663/0006-8101(2002)068[0038:00TBOT]2 $0 . \mathrm{CO} ; 2$

Yepez J. 2017. Ecología del género Puya (Bromeliaceae) en páramos del Volcán Chiles, de la Reserva Ecológica "El Ángel” y la Reserva Biológica "La Bretaña”, Andes orientales y occidentales del norte, Carchi-Ecuador. Tesis Magister. Escuela de Ciencias Biológicas. Facultad de Ciencias Exactas y Naturales. Pontificia Unidad Católica del Ecuador. Quito, Ecuador. http://repositorio.puce.edu.ec/handle/22000/13212

\section{Agradecimientos / Acknowledgments:}

Al personal del Herbario PRG-Lambayeque por facilitar la consulta y la revisión de las colecciones; a César Sánchez por el apoyo en las observaciones en campo y a Omar Mejía por la elaboración del mapa de colectas.

\section{Conflicto de intereses / Competing interests:}

Los autores no incurren en conflictos de intereses.

\section{Rol de los autores / Authors Roles:}

JEAV: revisión taxonómica del material de herbario, la redacción del manuscrito e ilustraciones. AMJC: redacción del manuscrito, generación de coordenadas y mapas, la categorización de especies. JEP: búsqueda de información bibliográfica, revisión de material de herbario.

\section{Fuentes de financiamiento / Funding:}

Los autores declaran que este trabajo no recibió ninguna financiación específica.

\section{Aspectos éticos / legales; Ethics / legals:}

Los autores declaran que no violaron ni omitieron normas éticas o legales en esta investigación. 
Página en banco

Blank page 